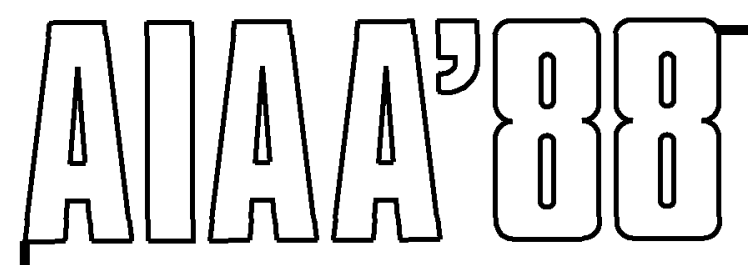

AIAA-88-2024

eas rement of eading dge Vortices

From a elta Wing Using a

hree omponent aser Velocimeter

$\mathrm{J}$ a $\mathrm{m}$ es $\mathrm{F}$. M e yers

NASA Langley R esearch C enter

$\mathrm{H}$ a m p to n, VA

Timothy E. Hepner

United $S$ tates Arm y ( AVRADCOM)

$\mathrm{H}$ a m pton, VA

AIAA 15th Aerod namic esting onference

$\mathrm{M}$ a y $18-20,1988 / \mathrm{S}$ a $\mathrm{D}$ iego, C A

Forpermission to copy or republish, contact the American Institute of A eronautics and Astronautics 370 L'Enfant Promenade, S.W., W a shington, D C 20024 


\title{
Measurement of Leading Edge Vortices from a Delta Wing Using a Three Component Laser Velocimeter
}

\author{
by \\ James F. Meyers \\ NASA - Langley Research Center \\ Hampton, Virginia 23665 \\ and \\ Timothy E. Hepner \\ United States Army (AVRADCOM) \\ Hampton, Virginia 23665
}

\begin{abstract}
A demonstration of the capabilities of a three component laser velocimeter to provide a detailed experimental database of a complex flow field is presented. The orthogonal three component laser velocimeter was used to measure the leading edge vortex flow field above a $75^{\circ}$ delta wing at angles-of-attack of $20.5^{\circ}$ and $40.0^{\circ}$. The resulting mean velocity and turbulence intensity measurements are presented. The laser velocimeter is described in detail including a description of the data processing algorithm. A full error analysis was conducted and the results presented.
\end{abstract}

\section{Introduction}

Classic aircraft design has been based on experimental results from wind tunnel models and free flight testing of prototype aircraft. Recent advances in computational fluid dynamics (CFD) are changing this approach. The aeronautical research community is beginning to depend more on the computations than on experimental results, to the point where predictions of flow field behavior are being used without supporting data. The question now becomes: Do these predictions of complex flow fields actually represent the real world? The present study is an attempt use a wind tunnel and state-of-the-art instrumentation to look into the real world. The object of this study is a common, yet complex flow - vortex rollup along the leading edge of a highly swept delta wing. 
A leading edge vortex pair contains a great deal of energy which, if carefully controlled, can provide significant lift on an aircraft. However, if a vortex is disturbed it will burst dissipating its energy in a random manner resulting in a sudden loss of lift. This problem is of special concern for aircraft stability when only one vortex of a coupled pair bursts, resulting in sudden substantial rolling moments being applied to the aircraft.

The subject of the present investigation is the flow above a $75^{\circ}$ delta wing. The Basic Aerodynamics Research Tunnel (BART) has been selected as the approximation of the real world. This wind tunnel has a very low turbulence in put flow which remains stable for the long periods of time required to conduct detailed investigations. An orthogonal three component laser velocimeter serves as the primary measurement technique. The resulting mean and turbulent intensity statistics from investigations of burst and unburst vortex flow fields above the delta wing are presented.

\section{Nomenclature}

$L \quad$ centerline length of $75^{\circ}$ delta wing model $(0.569 \mathrm{~m})$

$q$ dynamic pressure, $\rho \mathrm{V}_{\infty}{ }^{2} / 2$

$U \quad$ velocity component in the streamwise direction, $\mathrm{m} / \mathrm{sec}$

$V \quad$ velocity component in the vertical direction, $\mathrm{m} / \mathrm{sec}$

$V_{\infty} \quad$ freestream velocity, $\mathrm{m} / \mathrm{sec}$

$W \quad$ velocity component in the traverse direction, $\mathrm{m} / \mathrm{sec}$

$x \quad$ distance in the streamwise direction, $\mathrm{m}$

$\alpha \quad$ model angle-of-attack, deg

$\rho$ density, $\mathrm{Kg} / \mathrm{m}^{3}$

\section{Test Apparatus}

Undertaking a definitive experimental investigation of a very complex, highly turbulent flow field places severe requirements on the test facilities. The wind tunnel must provide a very stable flow field that maintains a constant speed with very low input turbulence. These 
requirements are critical in this investigation since the vortices must remain stable in strength and position during the long test times. The model must be of sufficient strength to minimize bending and mounted on a support with sufficient rigidity to minimize vibrations.

\section{Wind Tunnel}

The NASA Langley Basic Aerodynamic Research Tunnel ${ }^{1}$ shown in Figure 1 is an open circuit tunnel that has a test section measuring 0.71 meters high, 1.02 meters wide and 3.05 meters long. The test section is divided into two 1.524 meter long bays. The maximum flow velocity in the test section is $67 \mathrm{~m} / \mathrm{sec}$ which yields a Reynolds number of 4.6 million per meter. The airflow entering the test section is conditioned by a honeycomb, four anti-turbulence screens and an 11:1 contraction ratio. The 0.1 meter thick honeycomb has a 0.01 meter cell size. The screens are 7.87 mesh per centimeter with a porosity (ratio of open area to total area) of 64 percent. The tunnel is powered by a 125 horsepower AC motor coupled to a magnetic clutch. An electronic speed controller maintains the fan rpm within 0.1 percent of full scale (less than $1 \mathrm{rpm}$ variation).

These flow conditioners coupled with an excellent fan speed controller, provide a low-turbulence, uniform flow in the test section. The variation in the longitudinal component of turbulence intensity (measured with a hot wire) with test section $q$ is from approximately 0.05 percent at $q=4.13 \mathrm{~N} / \mathrm{m}^{2}(V=28 \mathrm{~m} / \mathrm{sec})$ to 0.08 percent at $q=18.6 \mathrm{~N} / \mathrm{m}^{2}(V=59.4 \mathrm{~m} / \mathrm{sec})$.

Optical access for the laser velocimeter is provided by large windows in the two walls and ceiling of both bays. The bay used for the laser velocimeter investigations has $11 \mathrm{~mm}$ thick optical glass windows and the remaining bay has Plexiglas windows. The windows can be exchanged between bays if necessary for test requirements.

\section{Model}

The model is a $75^{\circ}$ swept delta wing with a 0.305 meter span and sharp leading edges $\left(10^{\circ}\right.$ bevel on the lower surface with the upper surface flat). An $\alpha$ of $20.5^{\circ}$ yields a stable vortex flow above the model. An increase to an $\alpha$ of $40.0^{\circ}$ yields burst vortices. The tunnel speed is adjusted to obtain a chord Reynolds number of 1.0 million for both conditions. The investigations were conducted in a plane orthogonal to the surface of the model at an $x / L=0.7$. 


\section{Laser Velocimeter}

The laser velocimeter is chosen as the primary measurement system for these tests since it is non-intrusive and will not disturb the vortex flow field. The real time three component measurement capability allows the measurement of large turbulence intensities including reversed flows. These characteristics make the laser velocimeter unique among flow measurement techniques and ideal for obtaining definitive experimental databases for CFD verification.

\section{Optical Configuration}

While two component configurations using color separation are the accepted standard, three component designs are not as well established. The three component configurations currently suggested include various techniques from reference beam to off-axis fringe systems to measure the third or traverse component. A study of these various techniques ${ }^{2}$ indicates that they yield large increases (up to a factor of three) in turbulence intensity measurements with corresponding increases in statistical uncertainties in the computed mean of the traverse component. The greatest accuracy occurs when the third component measures the traverse component directly, that is, orthogonal to the remaining components. Therefore, an orthogonal three component system with color separation is chosen as the basic optical configuration.

The Argon ion laser wavelengths, $496.5 \mathrm{~nm}, 476.5 \mathrm{~nm}$ and $514.5 \mathrm{~nm}$, are chosen for the $U$ (streamwise), $V$ (vertical), and $W$ (traverse) components respectively. The transmission optical system for the $U$ and $V$ components is located on the side of the test section with the receiving optical system located above the test section slightly offperpendicular $\left(15^{\circ}\right)$ toward the upstream direction. The transmission optical system for the $W$ component is placed above the test section

perpendicular to the $U-V$ optical axis. Its receiving optical system is adjacent to the $U-V$ transmitter, rotated $15^{\circ}$ downstream about the sample volume. This configuration yields a very small spherical sample volume, $140 \mu \mathrm{m}$ in diameter, and reduces the amount of scattered light or flare from the model surface entering the collecting optical system. The sample volume can be placed within $70 \mu \mathrm{m}$ from the unprepared model surface before flare reduces signal-to-noise below acceptable limits for the signal processing instrumentation. All three optical components contain Bragg cells to provide full measurement directionality. Full specifications for the laser velocimeter are listed in table I. 
A major concern in the design of a system with the receiving optics placed perpendicular to the transmitting optics is the loss of scattering efficiency from the seeding particles. A laser velocimeter simulation computer code 3 predicted the performance properties of various configurations for the three components. The resulting transfer functions for the final design (table I) as a function of seed particle size are illustrated in Figure 2. These results indicate the system should provide acceptable performance with polystyrene latex particles approximately $0.9 \mu \mathrm{m}$ in diameter. Experimental studies with the final optical system using $0.89 \mu \mathrm{m}$ diameter polystyrene yielded average signal levels of $0.2 \mathrm{~V}$ peak-to-peak in each component.

\section{Traversing Mechanism}

The optical system and Argon ion laser are placed on a three component mechanical traversing system. The traversing mechanism surrounds the test section providing the maximum number of optical mounting locations for flexibility of optical configuration designs for future test requirements. The mechanism provides a traversing range of one cubic meter with a resolution of $0.01 \mathrm{~mm}$ in each axis. The optical system and laser move as a unit driven by two slaved stepper motors in each of the two horizontal directions and by four slaved stepper motors in the vertical direction. The stepper motors are controlled by the data acquisition minicomputer and the position of each component is determined from shaft encoders placed on each axis. The traversing mechanism is shown installed around the BART test section in Figure 1 with a close-up of the system in Figure 3.

\section{Seed Particles}

A signal burst is obtained when a small seed particle passes through the optical fringe pattern established when two coherent laser beams cross. The optical considerations require a particle with a high index of refraction and large diameter, Figure 2. Yet aerodynamic considerations require light particles with small diameters. The aerodynamic restrictions for swirling flows as reported by Dring and $\mathrm{Suo}^{4}$ indicate that particles with Stokes numbers less than 0.01 will follow the circular streamlines of a free vortex.

The choice of seed particles varies from liquids such as DOP, kerosene, mineral oil, etc. to solids such as polystyrene latex, kaolin, etc. ${ }^{5}$. All liquid and most solid particles exhibit polydisperse size distributions. While the smaller particles have low Stokes numbers and will follow the flow, they will not scatter sufficient light to yield measurable signals. 
They do contribute, however to the background light level and thus lower the signal-to-noise ratio of signals from larger particles. This increases the minimum measurable particle size necessary to obtain a sufficient signal amplitude to compensate for the increased noise. The larger particles in the distribution have large Stokes numbers and will not follow the flow field, yet have a high probability of being measured because the laser velocimeter transfer function favors the larger particles. These characteristics result in bias errors in the measured mean velocities dependent on the severity of traversed flow gradients. The differing responses of the various particles to the gradients also result in artificial increases in measured turbulence intensities. ${ }^{6}$ Although techniques such as placing impactors in-line with the particle generator can reduce the occurrences of larger particles, the smaller noise producing particles will remain. A better method is to use a monodisperse particle such as polystyrene latex.

Polystyrene latex has a relatively high index of refraction, 1.59 , and yet has a specific gravity of only 1.002 . Thus the conflicting requirements of high optical scattering efficiency and small size may be satisfied. From Figure 2, a particle $0.9 \mu \mathrm{m}$ in diameter will scatter sufficient light to be detectable in all three components. This particle size also has a Stokes number less than 0.01 which will yield a tracking fidelity sufficient to follow the streamlines of a free vortex.

Particles $0.89 \mu \mathrm{m}$ in diameter are fabricated at NASA using the technique described by Nichols ${ }^{7}$. They are suspended in a mixture of ethanol and water and injected in to the flow upstream of the honeycomb using an atomizing spray nozzle. The spray nozzle is mounted on a two component mechanical traversing system controlled by the tunnel data acquisition computer to keep the particle streamline passing through the laser velocimeter sample volume. The ethanol and water evaporate leaving the dry polystyrene to pass through the laser velocimeter sample volume. No evidence of particle agglomeration has been found based on oscilloscope observations of the signal bursts.

\section{System Electronics}

The system electronics consist of the photomultiplier tubes, line driving amplifiers, Bragg cell downmix circuitry, signal processors, data acquisition hardware and controlling minicomputer. The photomultipliers are side window type with a quantum efficiency of 21 percent and have $2.0 \mathrm{nsec}$ single photon rise times. Photoelectron amplification is by an 11 stage dynode chain and an external linedriving amplifier yielding a total gain of approximately 2 million. The output signal from each channel's line driver is passed through its 
respective downmix circuit to lower the $40 \mathrm{MHz}$ Bragg bias frequency to $10 \mathrm{MHz}$.

The downmixed signals are input to high-speed burst counters with a maximum reference clock frequency of $500 \mathrm{MHz}$. Signal verification is based on double-threshold detection and 5:8 count comparison accuracy checks. The measured data are transferred to the laser velocimeter autocovariance buffer in terface (LVABI) ${ }^{8}$ for temporary storage during the acquisition cycle. The LVABI acquires data from the three components simultaneously with a maximum data acquisition rate of 600,000 per second per channel. Although the total data storage per channel is $64 \mathrm{k}$ words, statistical measurements are typically limited to acquisition ensembles of 4096 words. Each velocity acquisition channel has a companion interarrival time channel that measures the time between successive velocity measurements. These interarrival time measurements are temporarily stored within the channel's memory. When the desired number of measurements is acquired or the acquisition time (typically one minute) has elapsed, the acquired velocity and interarrival time data for each velocity component are transferred via direct memory access to the host minicomputer for online processing and storage.

\section{Data Processing}

The processing of the laser velocimeter data evolves through several stages. The acquired data are transferred from the LVABI to the host minicomputer upon completion of the acquisition cycle. The high-speed burst counter data are decoded to obtain the number of reference clock pulses counted during the passage of each particle through the sample volume. The ensemble is examined to remove erroneous data and the interarrival times adjusted to compensate for the removed data. The velocity time histories are analyzed to determine the approximate integral time scale of the flow which is required to remove any effects of sampling bias. The resulting velocity distributions are then analyzed to determine the measurement statistics. The raw and converted data along with the statistical results are stored on magnetic tape. The final stage is the display and printing of the numeric results and the plotting of color coded velocity vectors on the graphic display.

Once the acquired velocity data are transferred to the host minicomputer and decoded to yield an ensemble of reference clock counts, measurements of electronic noise are removed. These spurious measurements are an artifact of all high-speed burst counters and represent up to 1 percent of the acquired data. An assumption is made that these measurements are randomly distributed and well away from 
the true velocity measurements. The ensemble data are grouped by value in histogram format, and the reference clock count with the greatest number of occurrences is determined. A measurement acceptance threshold is established as 5 percent of the peak number of occurrences (a value equal to approximately three standard deviations if the distribution were Gaussian) with the minimum possible value being 3 . The envelope of the histogram is followed from the peak to the right until the envelope goes below the acceptance threshold for three consecutive bins. This is assumed to be the upper limit of the distribution. All data to the right of this limit are considered spurious and removed. This procedure is repeated on the left of the peak. As each spurious datum is removed from the component ensemble, the neighboring interarrival times are adjusted to maintain the integrity of time.

The remaining ensemble is integrated and compared with the original total. If the integral is less than 90 percent of the original, a bimodal distribution may exist. The original histograms are examined for secondary distributions outside the major distribution limits. If a secondary is found, it is segregated in the same manner as the major distribution and held as a second ensemble for later reduction.

Once the spurious and/or secondary data are removed, the data are combined with the measured interarrival times to develop a velocity time history. The minimum and maximum velocities are determined and used to establish the resolution or bin width of a 10 bin velocity histogram. The velocity divisions established by the histogram are overlaid on the velocity time history. The time history is then tracked to determine the average time the velocity record resides within a division. Assuming the change in velocity is small during an integral time scale of the flow, the average residence time may be considered an estimate of this scale. (Comparisons of time scale estimates obtained using this technique with time scales determined from simultaneous hot wire measurements of a fully developed turbulent pipe flow indicate these estimates are approximately 20 percent of each time scale.)

These integral time scale estimates are used in the method developed by Edwards and Meyers ${ }^{9}$ to insure an independent sampling of the velocity field. This technique is based on two suppositions: the velocity field is coherent during the integral time following a measurement and thus additional measurements occurring during this time yield no further information; and, the number of additional measurements yield an indication of the probability of occurrence of that measured velocity. The method is implemented by establishing two histograms, the normal velocity histogram of measured velocities and the second containing the number of additional measurements during the integral time scale 
following each measured velocity. The first measurement in the velocity time history is added to the velocity histogram in the appropriate bin. The estimated integral time scale is extended along the time history and the number of measurements occurring within this time determined. This number is added to the occurrence histogram in the bin determined by the first measurement placement in the velocity histogram. The next measurement following the time scale is the new first measurement and the procedure repeated.

When the entire time history is examined, the occurrence histogram is divided into the velocity histogram yielding a normalized velocity distribution with all sampling biases removed. For example, if the data rate rises with increased velocity for a uniformly seeded flow as proposed by McLaughlin and Tiederman ${ }^{10}$, the occurrence histogram will contain more entries in the higher velocity bins. Normalizing the velocity histogram will reduce the number of measurements of higher velocities thus removing the bias. If there is no correlation between data rate and velocity as found by Meyers and Wilkinson ${ }^{11}$ in the center of a turbulent pipe flow, the occurrence histogram will be flat with an equal number in each bin.

The final step in the on-line data processing is the storage of the data on magnetic tape in raw form and engineering units. The computed statistics for each component are also stored along with the tunnel free stream parameters and the location of the sample volume. The statistical results are displayed on the operator's terminal and printed on hard copy. The mean velocity vector is determined based on the component velocity means and displayed on a graphics terminal. The vertical and traverse components are used to determine the length and direction of the vector and the streamwise component is used to determine the color of the vector. These on-line displays provide the operator with both detailed test information and an overall look of the entire data set as testing progresses.

\section{Measurement Errors}

The laser velocimeter is susceptible to measurement inaccuracies as is any measurement device. The ultimate uncertainty in laser velocimetry is based on the ability of the seeding particle to follow the gas flow. If it does not, the measurements will be in error regardless of the capabilities of the instrumentation. Based on the study by Dring and $\mathrm{Suo}^{4}$ and the use of $0.89 \mu \mathrm{m}$ diameter polystyrene, the particles should track the flow within 1 percent. Instrumentation uncertainties include optical errors, signal processing uncertainties, and uncertainties in statistical estimations. 


\section{Optical Errors}

Measurement uncertainties due to the optical system include the uncertainty in the measurement of the cross beam angles, diverging fringes ${ }^{12}$, velocity gradients across the sample volume, and sample volume position uncertainties. The largest of these errors is the uncertainty in the measurement of the cross beam angle. The accepted technique is to measure the angle geometrically. A focal distance of 4.25 meters was chosen and an uncertainty of $\pm 0.5 \mathrm{~mm}$ is estimated in locating the center of each laser beam. This results in a bias error in the velocity measurements within the range of \pm 0.5 percent.

If the transmitted laser beams do not focus at the crossover, the light wavefronts will not be parallel as they cross. This results in a fringe pattern that is either diverging or contracting along the optical axis. The variation in fringe spacing will yield different signal frequencies for the same velocity depending where the particle passes through the sample volume. The output beam from the laser in the present system is collimated to insure that the crossing laser beams focus at the sample volume removing this error.

The remaining optical errors have the greatest significance in large velocity gradients. A severe velocity gradient will increase the measured turbulence intensity as the velocities vary along the length of the sample volume, and will translate a position uncertainty to an uncertainty in the mean velocity measurement. The sample volume in the present system is nominally a $150 \mu \mathrm{m}$ diameter sphere due to the orthogonal location of the receiving optical system. This will yield a difference of velocity across the sample volume of 0.4 percent at the edge of the vortex core where the velocity gradient of $100 \mathrm{~m} / \mathrm{sec} / \mathrm{cm}$ is the largest in the flow field. The location of the sample volume can be resolved to approximately $50 \mu \mathrm{m}$ based on the onset of flare from the model surface and the $10 \mu \mathrm{m}$ resolution of the traversing mechanism. This yields a worse case uncertainty in the mean velocity measurement of \pm 0.15 percent, again at the edge of the vortex core.

\section{Electronic Errors}

Errors due to the system electronics include photomultiplier bandwidth biasing, filter biasing, high-speed burst counter clock synchronization and quantizing, and signal-to-noise effects. The maximum signal frequency obtained during the present investigation is the $40 \mathrm{MHz}$ Bragg frequency plus the signal frequency for the maximum flow velocity of $70 \mathrm{~m} / \mathrm{sec}$ or $7.0 \mathrm{MHz}$. The resulting input signal frequency of $47 \mathrm{MHz}$ is far below the $250 \mathrm{MHz}$ bandwidth of the photomultiplier and 
the $200 \mathrm{MHz}$ bandwidth of the line driving amplifier. Thus limited photomultiplier bandwidth biasing is negligible. The output signal from the photomultiplier is mixed with a signal from the original Bragg cell oscillator to yield a downmixed center or effective Bragg frequency of $10 \mathrm{MHz}$. The measured velocity limits (based on three standard deviations about the mean) during the investigation are:

Streamwise

-47.62 to $70.34 \mathrm{~m} / \mathrm{sec} \rightarrow 6$ to $17 \mathrm{MHz}$

Filter setting 4 to $32 \mathrm{MHz}$

Vertical

-47.43 to $54.08 \mathrm{~m} / \mathrm{sec} \rightarrow 5$ to $16 \mathrm{MHz}$

Filter setting 4 to $16 \mathrm{MHz}$

Traverse

-66.04 to $43.98 \mathrm{~m} / \mathrm{sec} \rightarrow 4$ to $14 \mathrm{MHz}$

Filter setting 4 to $16 \mathrm{MHz}$

With filter settings wider than the extremes in signal frequencies (based on the maximum measurement mean plus three standard deviations and the minimum mean minus three standard deviations), the input signal amplitudes are unchanged, and thus filter bias is not a factor.

Calculations of the clock synchronization and quantizing errors found in high-speed burst counters represent errors for an infinite signal-tonoise ratio. The coupling of the degradation in signal-to-noise on these errors is not fully understood. Using a Monte Carlo simulation of the high-speed burst counter ${ }^{13}$ the effects of signal-to-noise may be examined. The input signal to the simulator was chosen to have a constant frequency of $10.0 \mathrm{MHz}$ with the amplitude varied from $0.02 \mathrm{~V}$ (700 photons/burst) to $0.2 \mathrm{~V}(10,500$ photons/burst) resulting in variations in the input signal-to-noise ratio. Histograms of the simulator output for 100 signal bursts at the various signal amplitudes are presented in Figure 4. At a signal level of $0.2 \mathrm{~V}$, the histogram shows the typical \pm 1 clock count uncertainty expected for quantizing error. As the signal amplitude drops, the histograms spread far beyond 
quantizing levels. The resulting statistical standard deviations are presented in Figure 5.

\section{Statistical Uncertainties}

As with any statistical estimate of a physical phenomenon, the statistics will have an uncertainty based on the standard deviation of the phenomenon about its average and the number of independent samples of that phenomenon. The procedure used to process the acquired velocity data yields a histogram based on independent samples of the measured velocity and may be used to estimate the mean, standard deviation and other higher order statistics of the velocity component at that point in the flow field. The number of measurements contained within this histogram is lost however. Therefore the number of independent measurements is approximated by the number of integral time scales contained within the data ensemble as determined from the measured interarrival times and the estimate of the time scale. The resulting statistical uncertainties are typically 1.0 percent reaching a maximum of 6.3 percent within the vortex core where the number of measurements is low due to the scarcity of particles and the long integral times.

\section{Flow Field Measurements}

The flow above the delta wing with an $\alpha$ of $20.5^{\circ}$ consists of two stable leading edge vortices. A laser light sheet visualization of these vortices is shown in Figure 6. In a companion paper by Sellers and Kjelgaard ${ }^{1}$ this flow field was studied using surface and laser light sheet visualizations, pitot pressure surveys, and 5-hole probe surveys. Theoretical estimates of the flow were computed using the CFL3D $\operatorname{code}^{14}$ which uses a thin-layer formulation of the Navier-Stokes equations. The experimental and theoretical results compared well with the laser velocimeter data in regions of low velocity gradients, but deviated near the core to differences of up to 35 percent. The laser velocimeter was the only technique that could obtain turbulence results and descriptions of the velocity flow field when the angle-of-attack was increased to $40.0^{\circ}$, bursting the vortices.

The three component mean velocity measurements obtained with the laser velocimeter at an $\alpha$ of $20.5^{\circ}$ are shown in Figure 7 and at $40.0^{\circ}$ in Figure 8. It is noted that the streamwise component increases to twice freestream velocity within the core of the vortex, but reverses when the vortices burst. The angular motion seems to change little in either case. The stability of the two flows are illustrated by the plots of integral time 
scale in Figures 9 and 10 respectively. The unburst vortices are very stable with long time scales while the burst case has short time scales over the entire region.

The measurements of flow turbulence intensity are presented for the streamwise component, Figures 11 and 12 , the vertical component, Figures 13 and 14, and the traverse component, Figures 15 and 16 . The streamwise component contains the maximum turbulence near the core of the vortices while the maximum for the other two components are outboard of the vortices. The total turbulence energy may be approximated by computing the square root of the average of the component variances and normalizing by free stream velocity. These results are shown in Figures 17 and 18.

Close examination of Figure 7 indicates the possibility of small secondary vortices located below and outboard of the primary vortices. This area was investigated with the laser velocimeter with greater detail. The resulting measurements, shown in Figure 19, not only show a secondary vortex but evidence of a tertiary vortex.

\section{Concluding Remarks}

The advancement of computational fluid dynamic codes is outpacing the experimental data used to guide and verify the codes. In many cases the required measurements are too difficult if not impossible to obtain with classic instrumentation. A demonstration of state-of-the-art instrumentation in the form of an orthogonal three component laser velocimeter has been presented. The system characteristics and data processing algorithms have been presented giving a complete description of the instrumentation system. The analysis of measurement uncertainties has shown mean velocity measurements to be accurate to within 0.5 percent and turbulence intensity measurements to be accurate above twice the residual value of 1 percent. Mean velocity and turbulence intensity contours of the vortex flow field above a $75^{\circ}$ delta wing at an $\alpha$ of $20.5^{\circ}$ are given along with contours of burst vortices when the $\alpha$ was raised to $40^{\circ}$. These results along with the estimates of integral time scale demonstrate the capabilities of laser velocimetry to obtain the detailed experimental data sets necessary to validate the computational codes. 


\section{References}

1. Sellers, W. L., III and Kjelgaard, S. O.: The Basic Aerodynamics Research Tunnel - A Facility Dedicated to Code Validation. AIAA 15th Aerodynamic Testing Conference, San Diego, California, paper AIAA-88-1997, May 18-20, 1988.

2. Meyers, J. F.: The Elusive Third Component. Proceedings of the International Symposium on Laser Anemometry, FED-Vol. 33, American Society of Mechanical Engineers, Miami, Florida, pp. 247-254, November 17-22, 1985.

3. Meyers, J. F. and Walsh, M. J.: Computer Simulation of a Fringe Type Laser Velocimeter. Proceedings of Project Squid Workshop on the Use of the Laser Velocimeter for Flow Measurements, Purdue University, pp. 471-510, March 27-29, 1974.

4. Dring, R. P. and Suo, M.: Particle Trajectories in Swirling Flows. Journal of Energy, Vol. 2, No. 4, July-August 1978.

5. Wind Tunnel Seeding Systems for Laser Velocimeters. NASA Langley Research Center, NASA CP-2393, March 19-20, 1985.

6. Meyers, J. F.; Hunter, W. W., Jr.; Reubush, D. E.; Nichols, C. E., Jr.; Hepner, T. E.; and Lee, J. W.: Performance Test of Laser Velocimeter System for the Langley 16-foot Transonic Tunnel. NASA TP-2502, December 1985.

7. Nichols, C. E., Jr.: Preparation of Polystyrene Microspheres for Laser Velocimetry in Wind Tunnels. NASA TM-89163, June 1987.

8. Cavone, A. A.; Sterlina, P. S.; Clemmons, J. I., Jr.; and Meyers, J. F.: A High Speed Buffer for LV Data Acquisition. Proceedings of the International Congress on Instrumentation in Aerospace Simulation Facilities, College of William and Mary, Williamsburg, Virginia, pp. 113-119, June 22-25, 1987.

9. Edwards, R. V. and Meyers, J. F.: An Overview of Particle Sampling Bias. Proceedings of the Second International Symposium on Applications of Laser Anemometry to Fluid Mechanics, Lisbon, Portugal, paper 2.1, July 2-5, 1984.

10. McLaughlin, D. K. and Tiedermann, W. G., Jr.: Biasing Correction for Individual Realization of Laser Anemometer Measurements in 
Turbulent Flow. The Physics of Fluids, Vol. 16, No. 12, pp. 20822088, 1973.

11. Meyers, J. F. and Wilkinson, S. P.: A Comparison of Turbulence Intensity Measurements Using a Laser Velocimeter and a Hot Wire in a Low Speed Jet Flow. Proceedings of the International Symposium on Applications of Laser-Doppler Anemometry to Fluid Mechanics, Lisbon, Portugal, paper 17.4, July 5-7, 1982.

12. Hanson, S.: Broadening of the Measured Frequency Spectrum in a Differential Laser Anemometer Due to Interference Plane Gradients. J. Phys. D: Appl. Phys., Vol. 6, No. 2, pp. 164-171, January 1973.

13. Meyers, J. F. and Stoughton, J. W.: Frequency Domain Laser Velocimeter Signal Processor. Proceedings of the Third International Symposium on Applications of Laser Anemometry to Fluid Mechanics, Lisbon, Portugal, paper 3.6, July 7-9, 1986.

14. Thomas, J. L.; Taylor, S. L.; and Anderson, W. K.: Navier-Stokes Computations of Vortical Flows Over Low Aspect Ratio Wings. AIAA paper 86-0207, January, 1987.

Table I. Laser velocimeter specifications

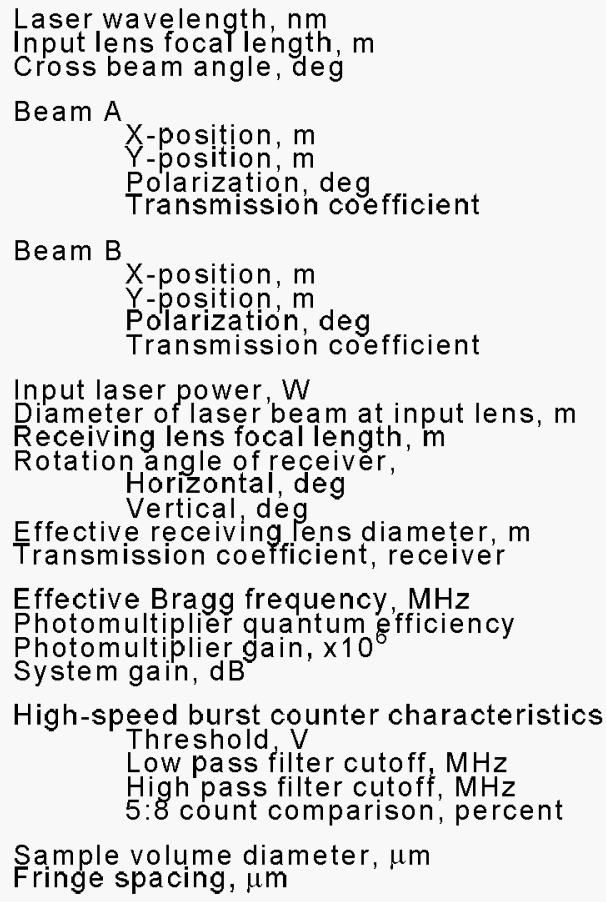

$\begin{array}{rrr}\text { streamwise } & \text { vertical } & \text { traverse } \\ 496.5 & 476.5 & 514.5 \\ 1.0 & 110 & 1.0 \\ 2.844 & 2.871 & 2.772 \\ & & \\ -0.025 & -0.025 & -0.025 \\ 0.0 & 0.0 & 0.0 \\ 90.0 & 0.0 & 0.0 \\ 0.4667 & 0.3960 & 0.3730 \\ & & \\ 0.025 & 0.025 & 0.025 \\ 0.0 & 0.0 & 0.0 \\ 90.0 & 0.0 & 0.0 \\ 0.3733 & 0.3960 & 0.4270 \\ 0.165 & 0.150 & 0.395 \\ 0.0049 & 0.0040 & 0.0040 \\ 1.0 & 1.0 & 1.0 \\ 90.0 & 90.0 & 90.0 \\ 75.0 & 15.0 & 15.0 \\ 0.062 & 0.062 & 0.062 \\ 0.744 & 0.728 & 0.741 \\ 10.0 & 10.0 & 10.0 \\ 0.21 & 0.21 & 0.21 \\ 2.0 & 10.0 & 2.0 \\ 10.0 & & 10.0 \\ & 0.0 & \\ 0.06 & 4.06 & 0.06 \\ 32.0 & 2.0 & 4.0 \\ 4.0 & 152.0 & 2.0 \\ 2.0 & 9.51 & 164.0 \\ 129.0 & & 10.64 \\ 10.0 & & \end{array}$




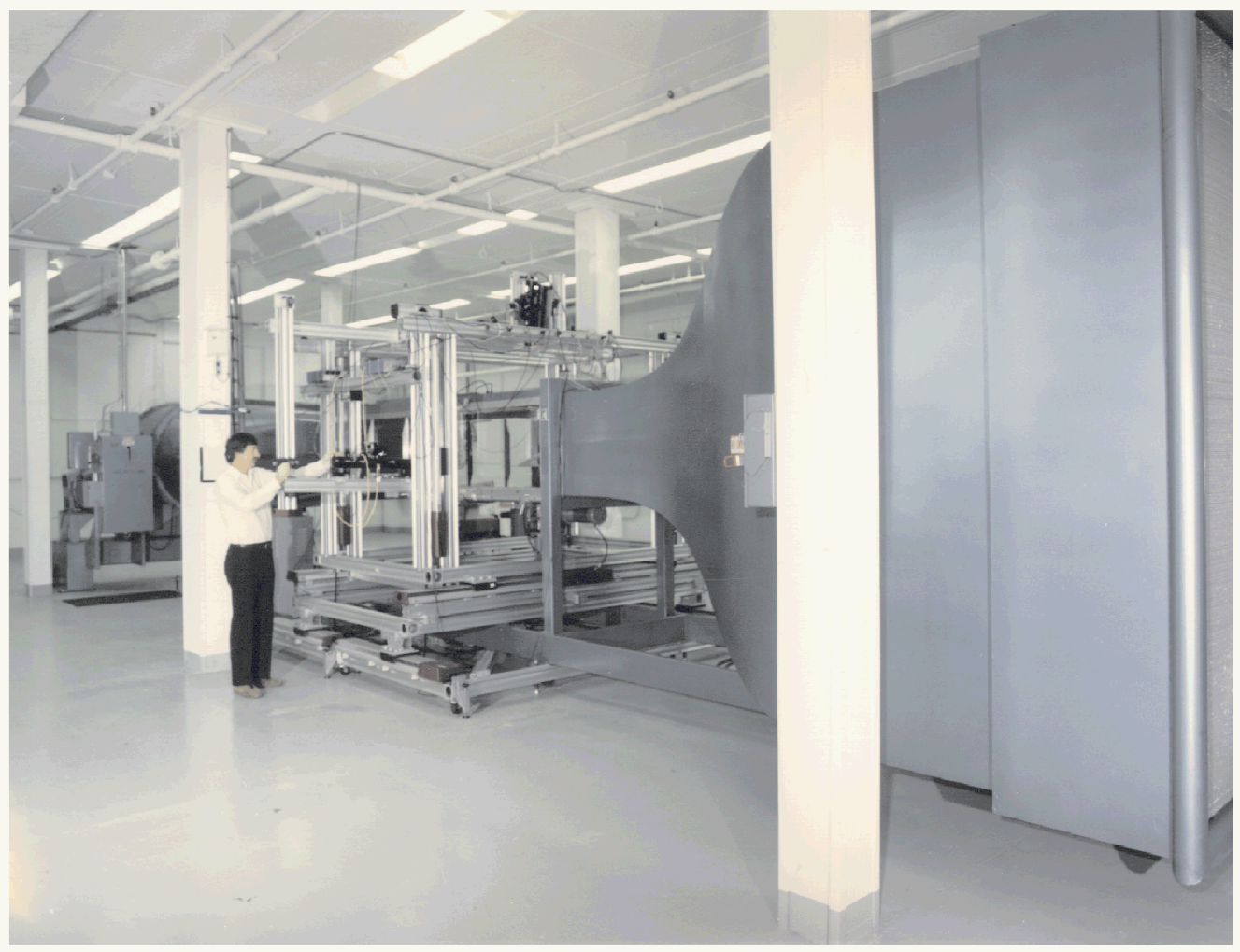

Figure 1.- NASA Langley Basic Aerodynamics Research Tunne1.

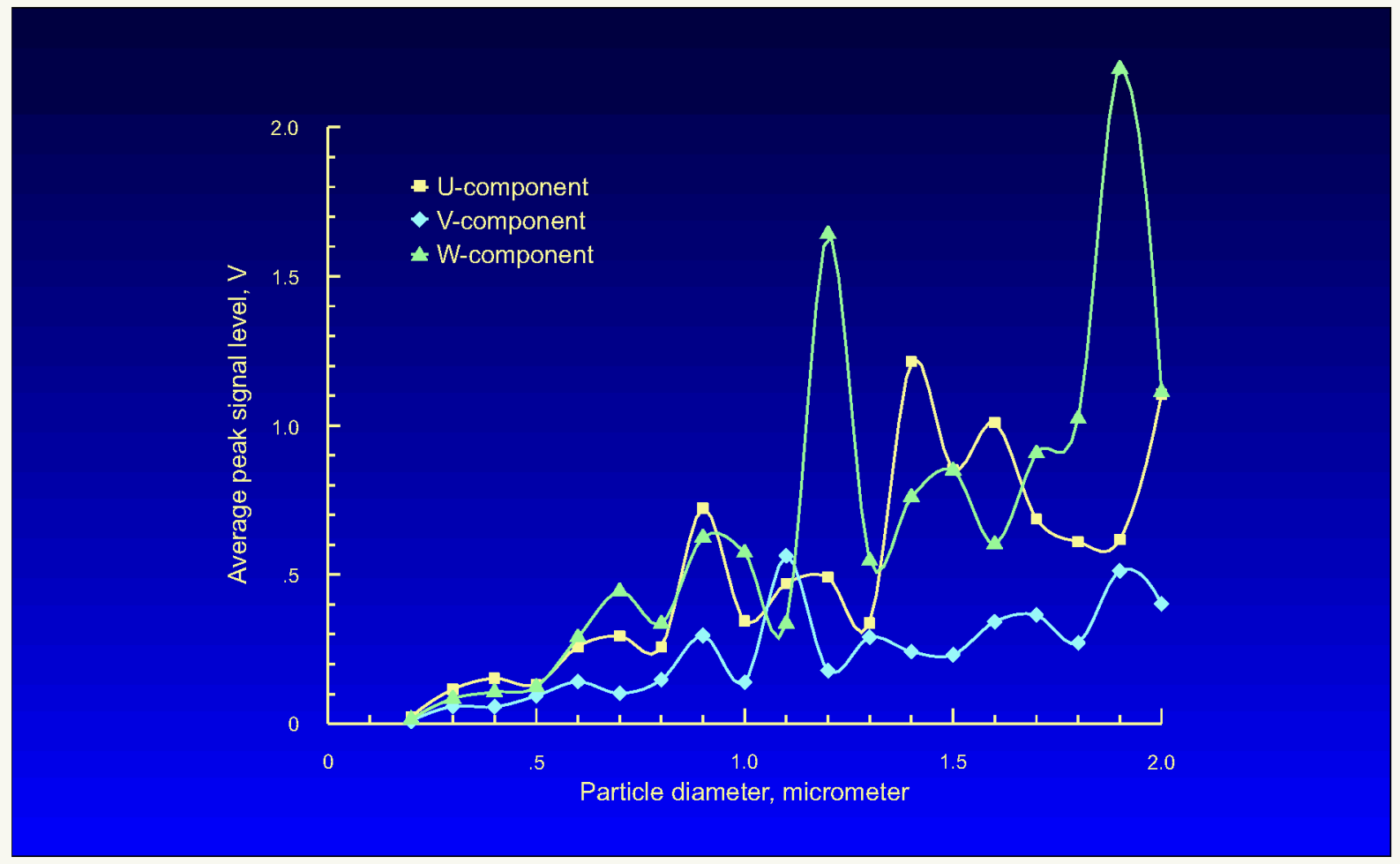

Figure 2.- Predicted laser velocimeter peak-to-peak signal level as a function of particle size for polystyrene latex. 


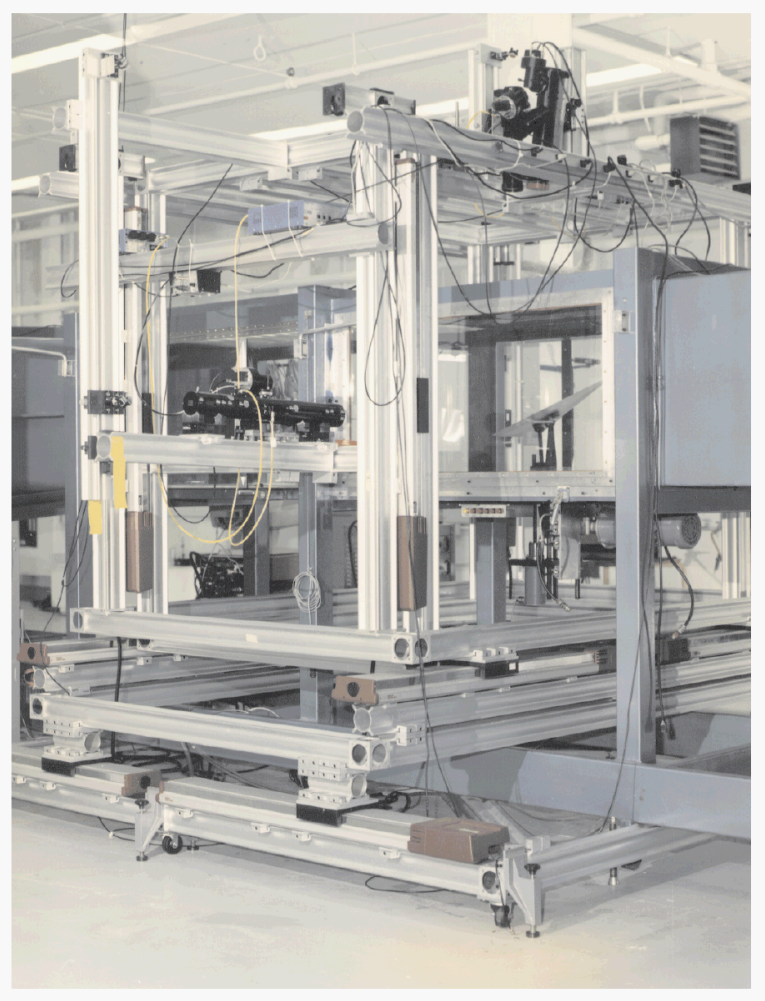

Figure 3.- Three component laser velocimeter mounted on the traversing mechanism.

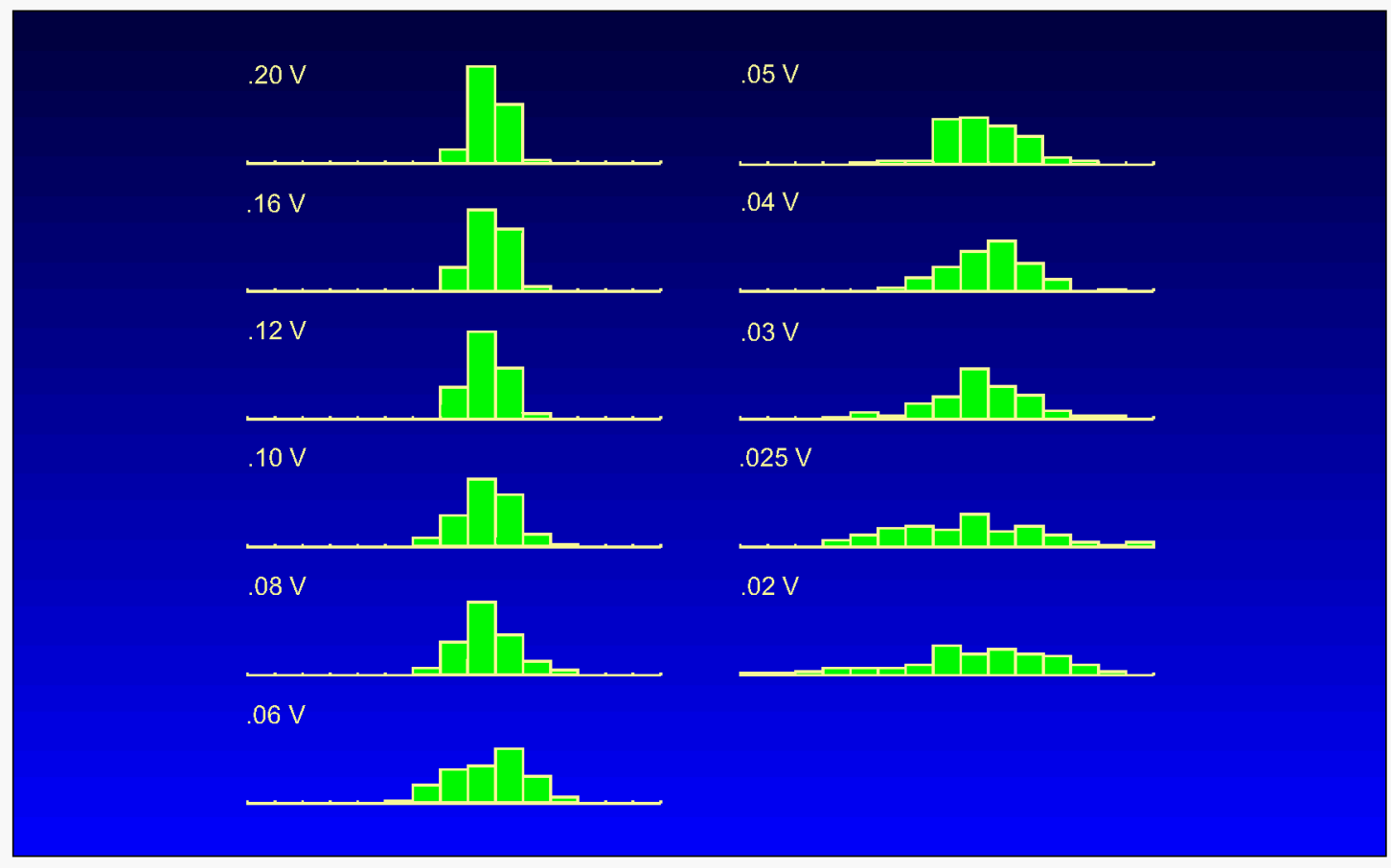

Figure 4.- Simulated histograms of high-speed burst counter output for various peak-to-peak signal amplitudes. 


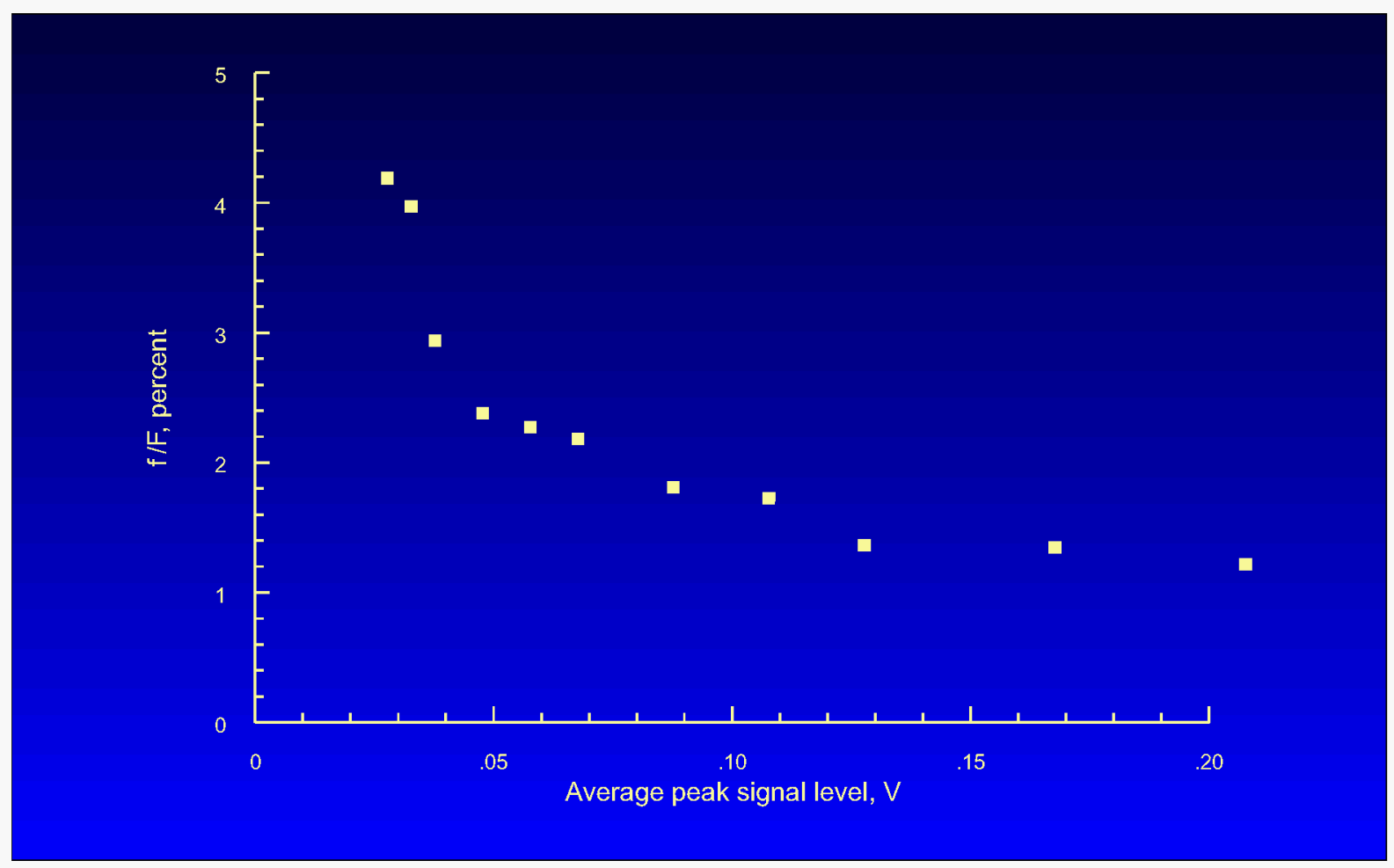

Figure 5.- Predicted statistical standard deviations of high-speed burst counter output for various peak-to-peak signal amplitudes.

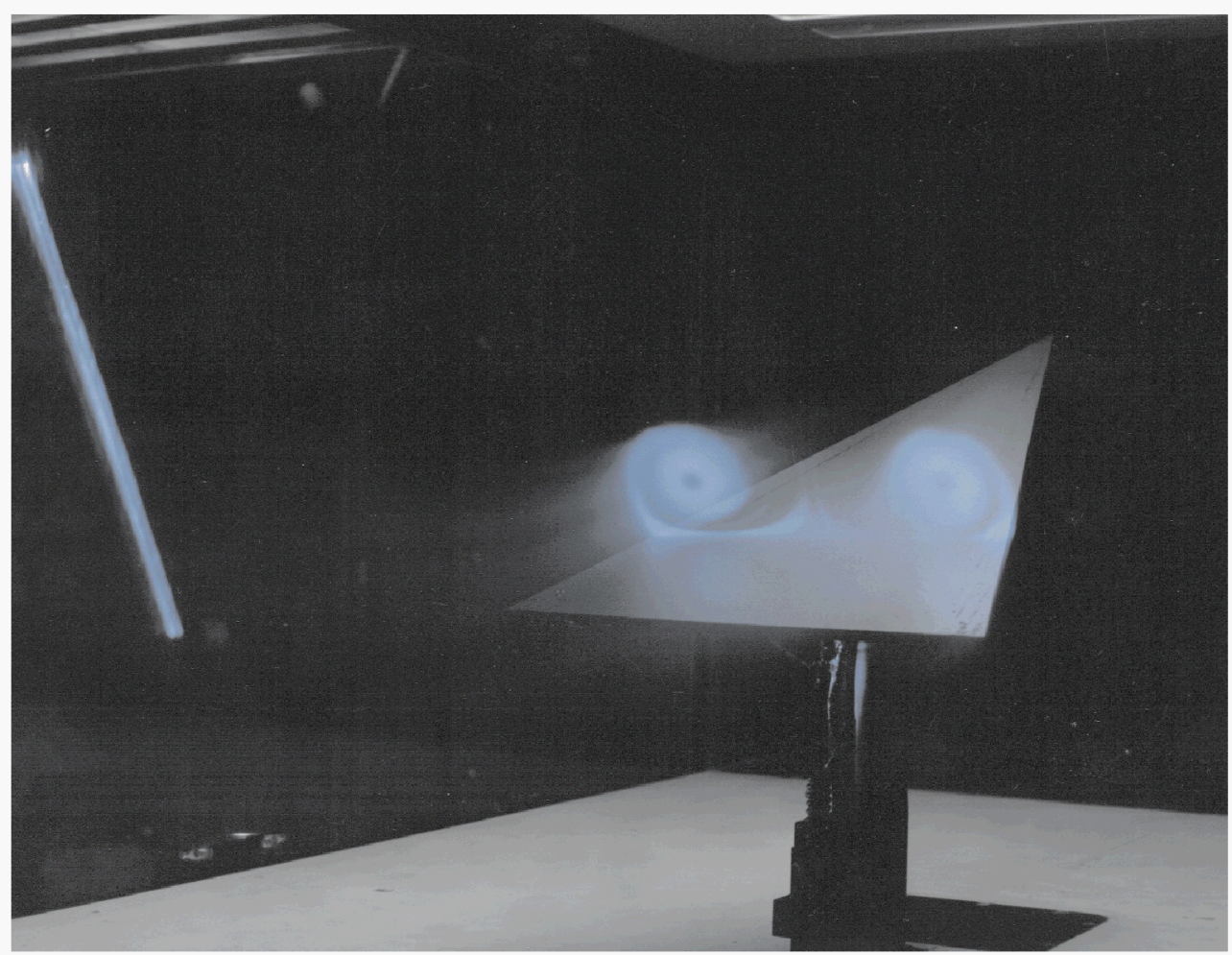

Figure 6.- Laser light sheet flow visualization of the vortex flow at an angle-ofattack $=20.5^{\circ}$. 


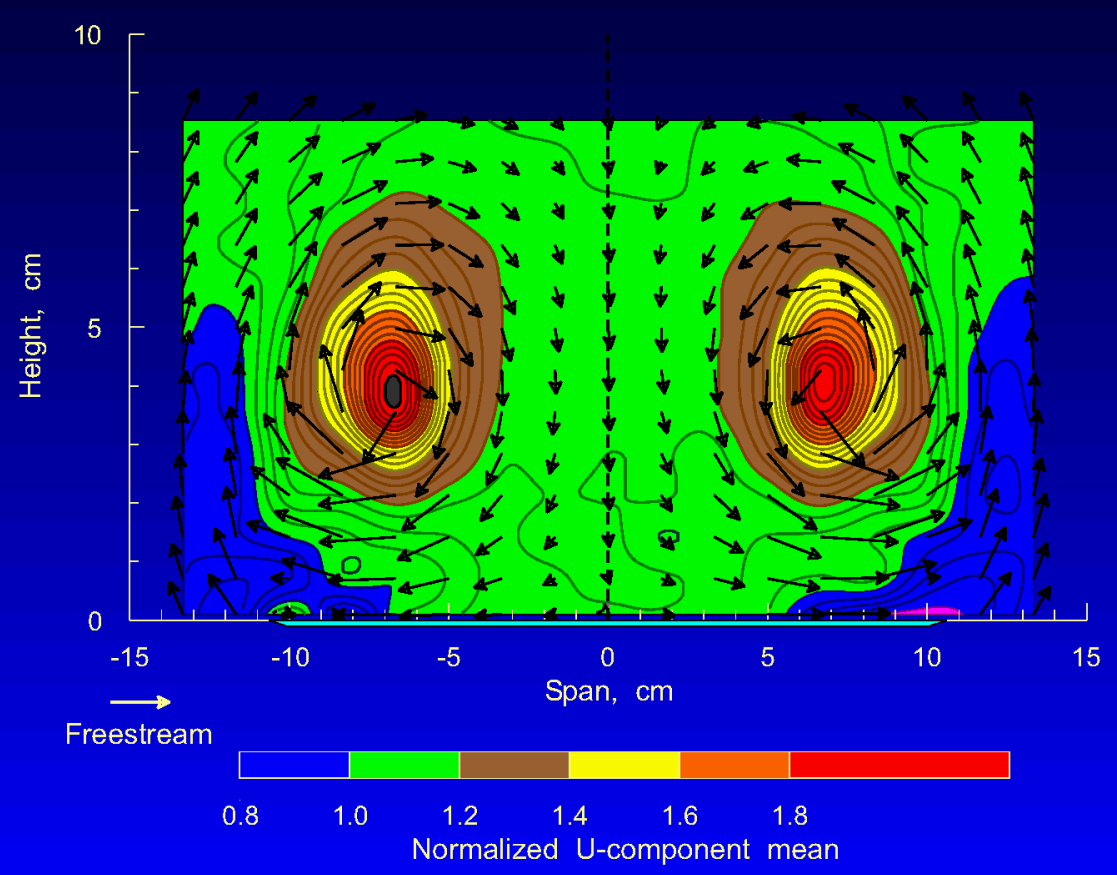

Figure 7.- Mean velocity measurements, $\alpha=20.5^{\circ}$.

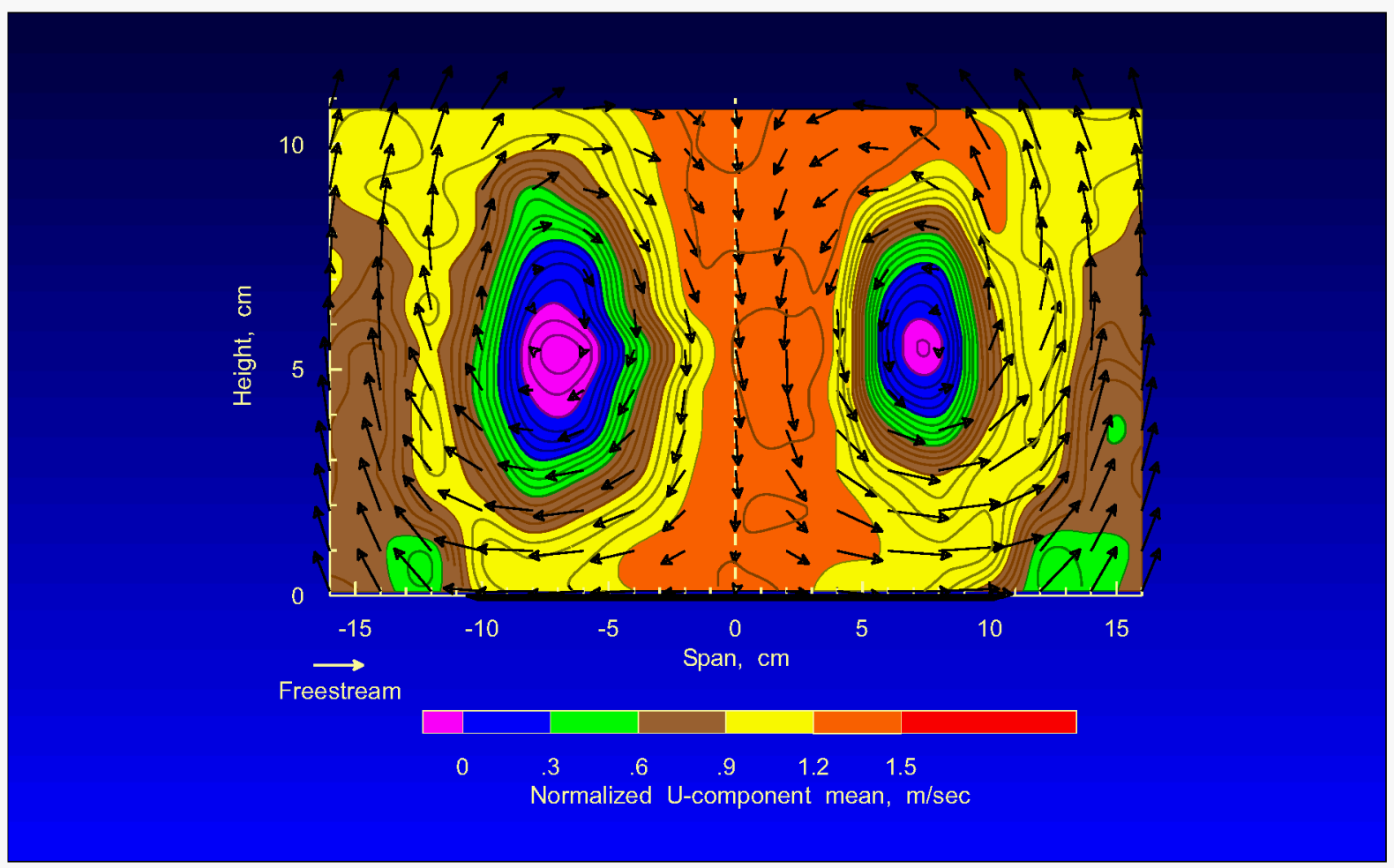

Figure 8.- Mean velocity measurements, $\alpha=40.0^{\circ}$. 


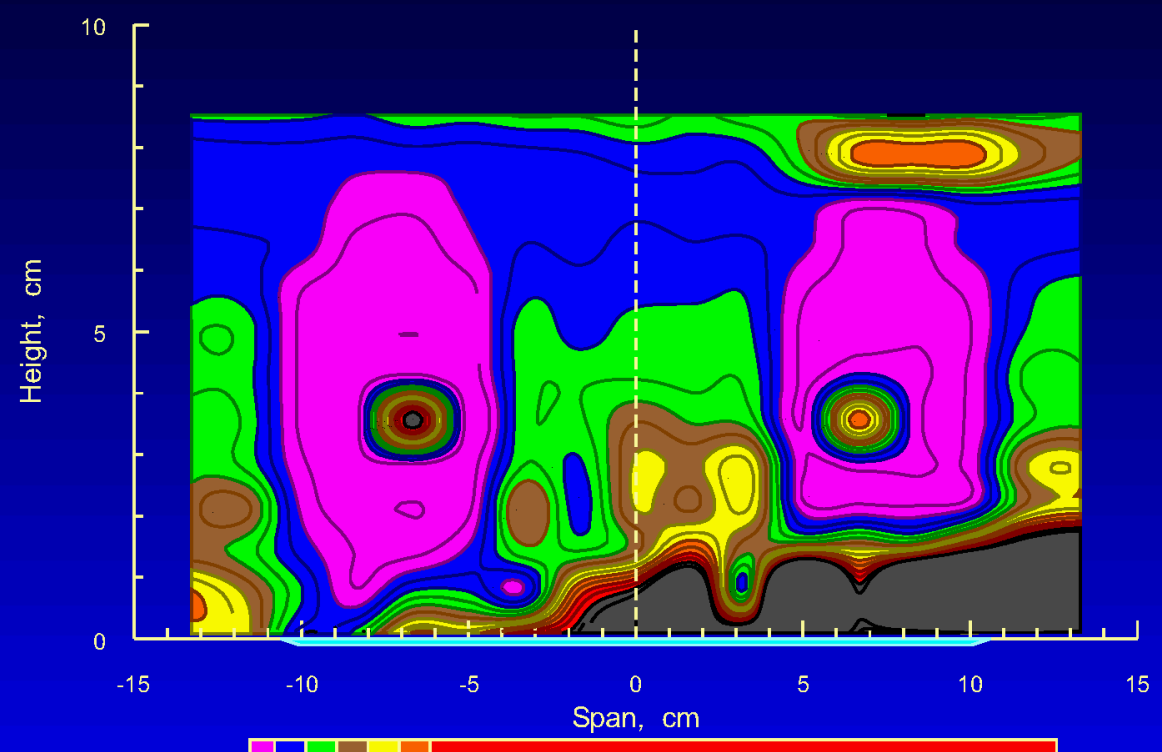

$\begin{array}{llllllll}1 & 2 & 3 & 4 & 5 & 5 & 6\end{array}$

Integral time scale, U-component, sec

Figure 9.- Contours of estimated integral time scales, $\alpha=20.5^{\circ}$

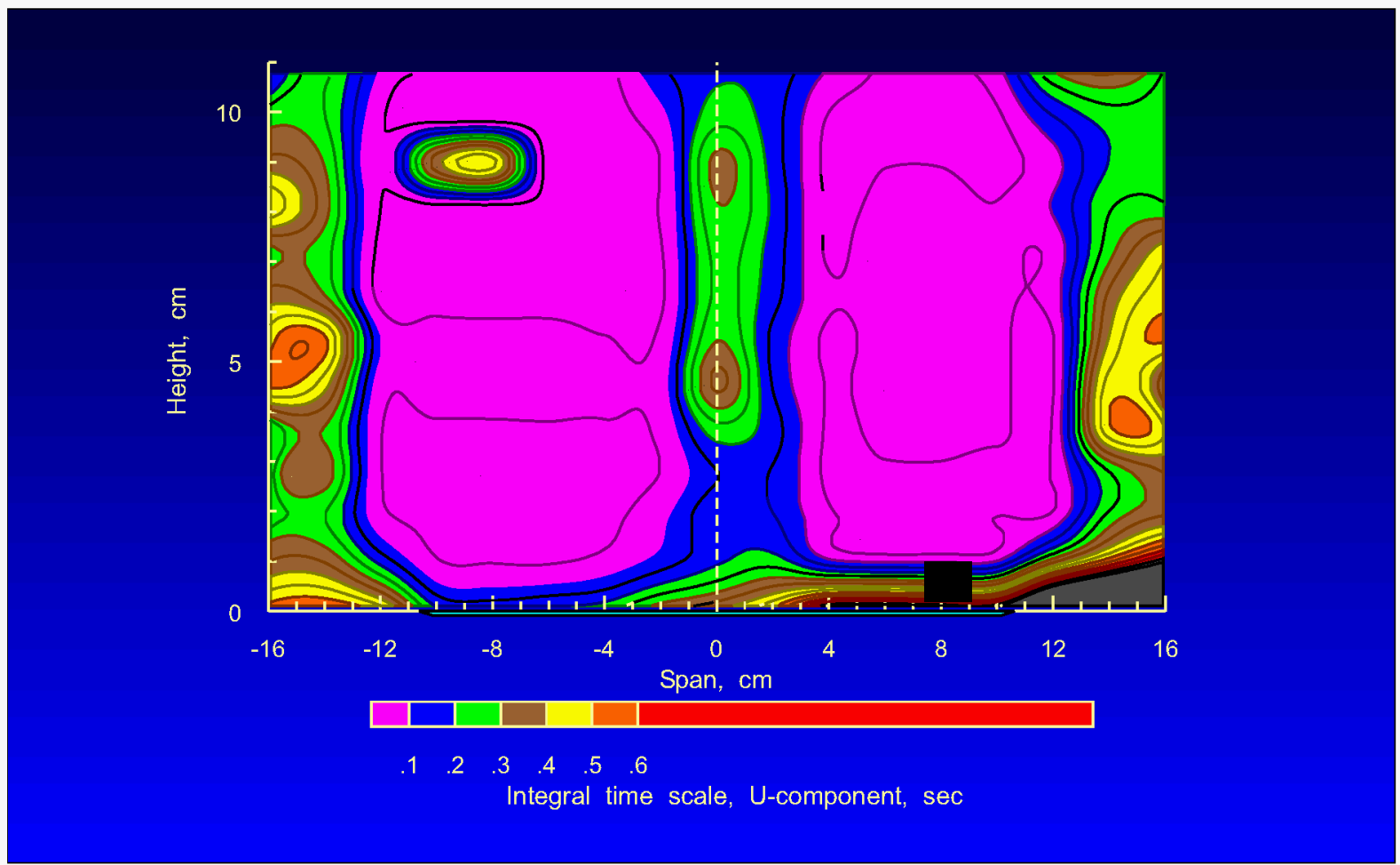

Figure 10.- Contours of estimated integral time scales, $\alpha=40.0^{\circ}$ 


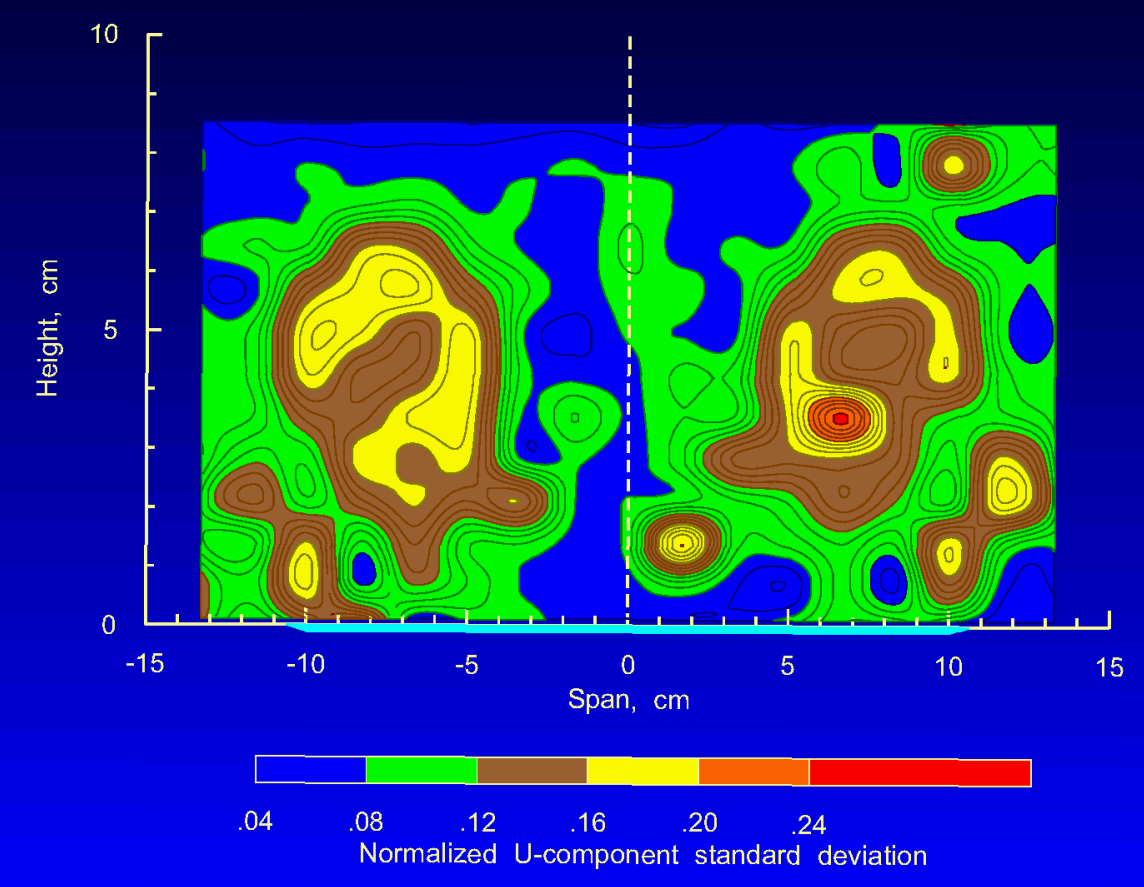

Figure 11.- Contours of streamwise turbulence intensity, $\alpha=20.5^{\circ}$.

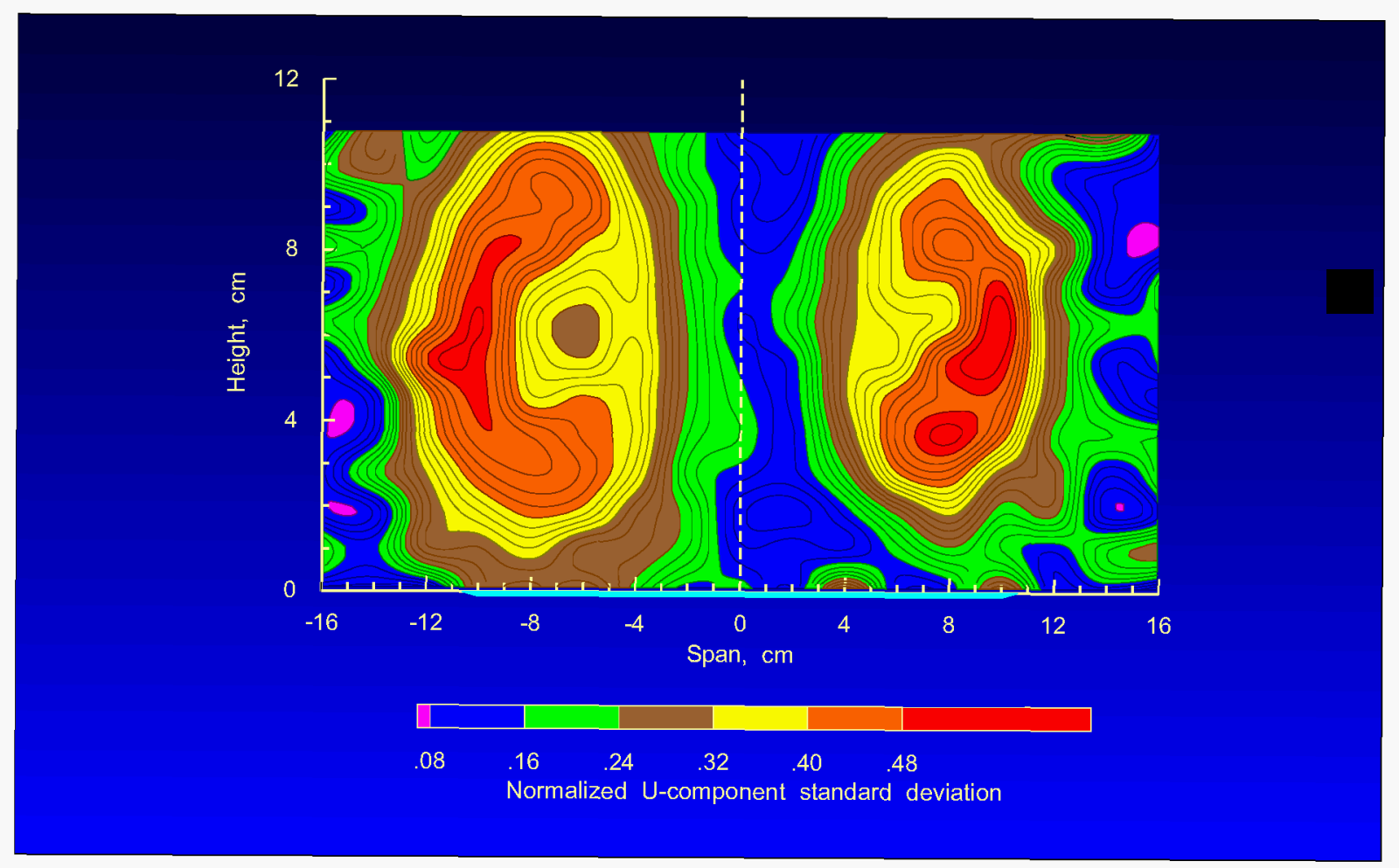

Figure 12.- Contours of streamwise turbulence intensity, $\alpha=40.0^{\circ}$. 


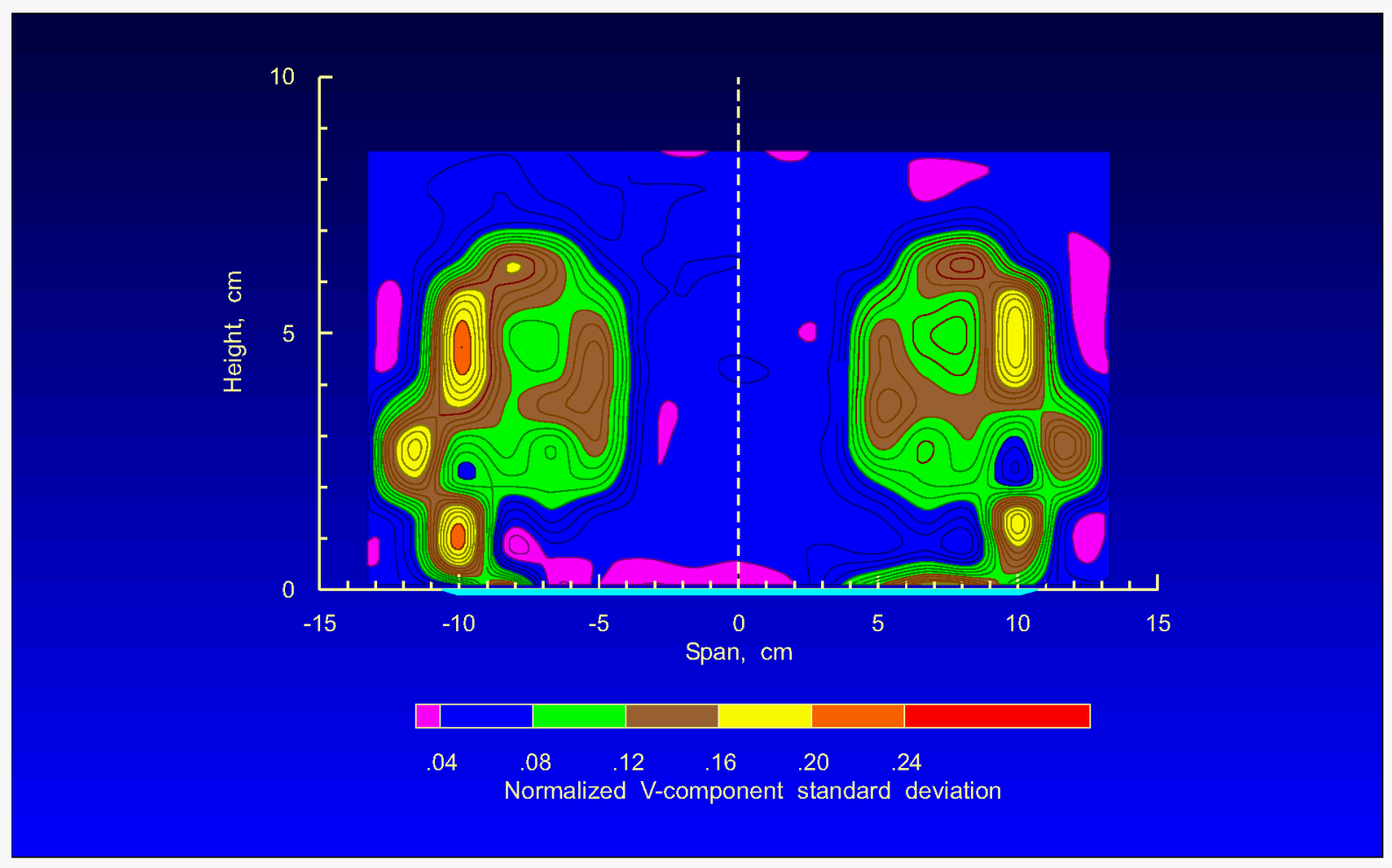

Figure 13.- Contours of vertical turbulence intensity, $\alpha=20.5^{\circ}$.

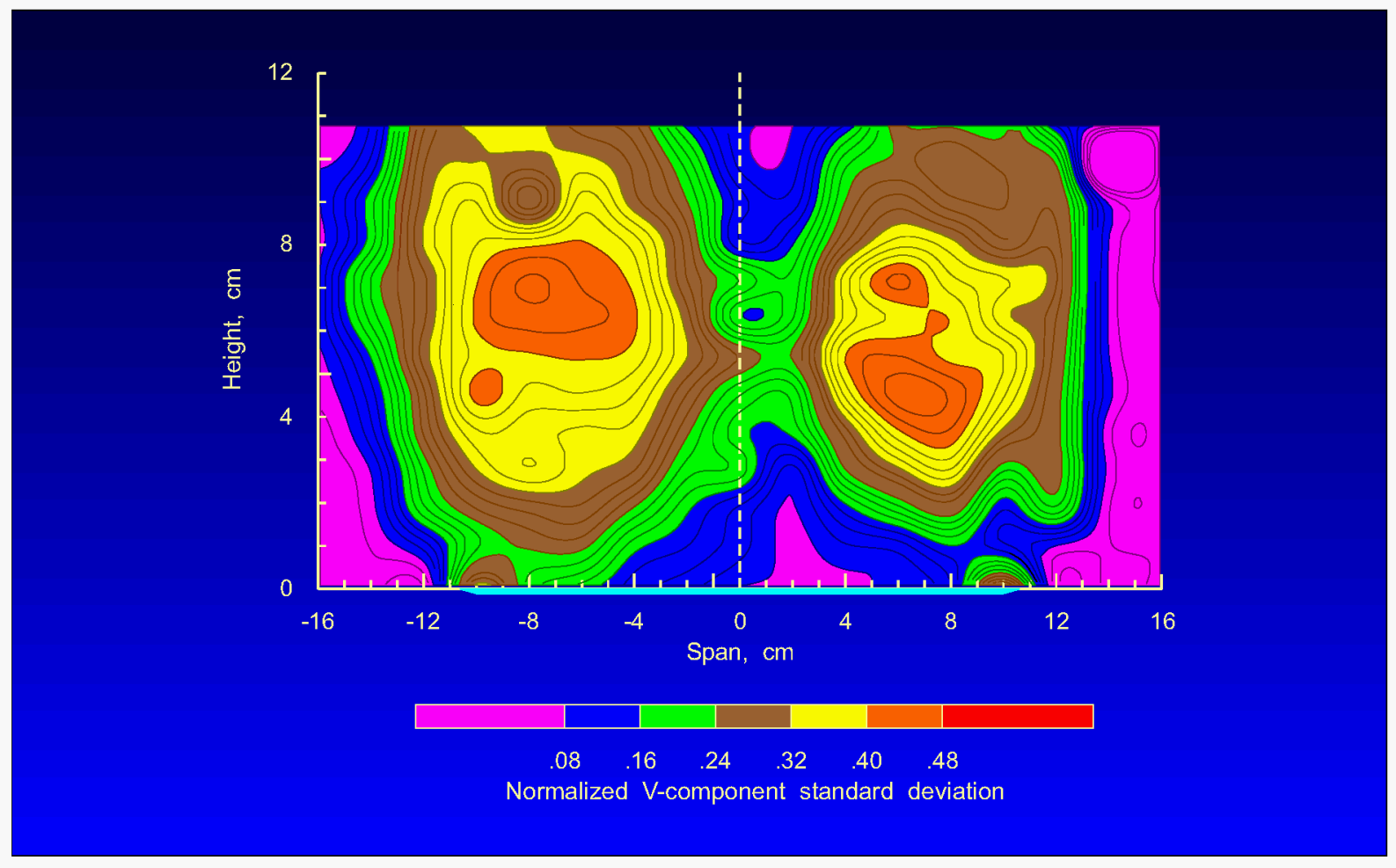

Figure 14.- Contours of vertical turbulence intensity, $\alpha=40.0^{\circ}$. 


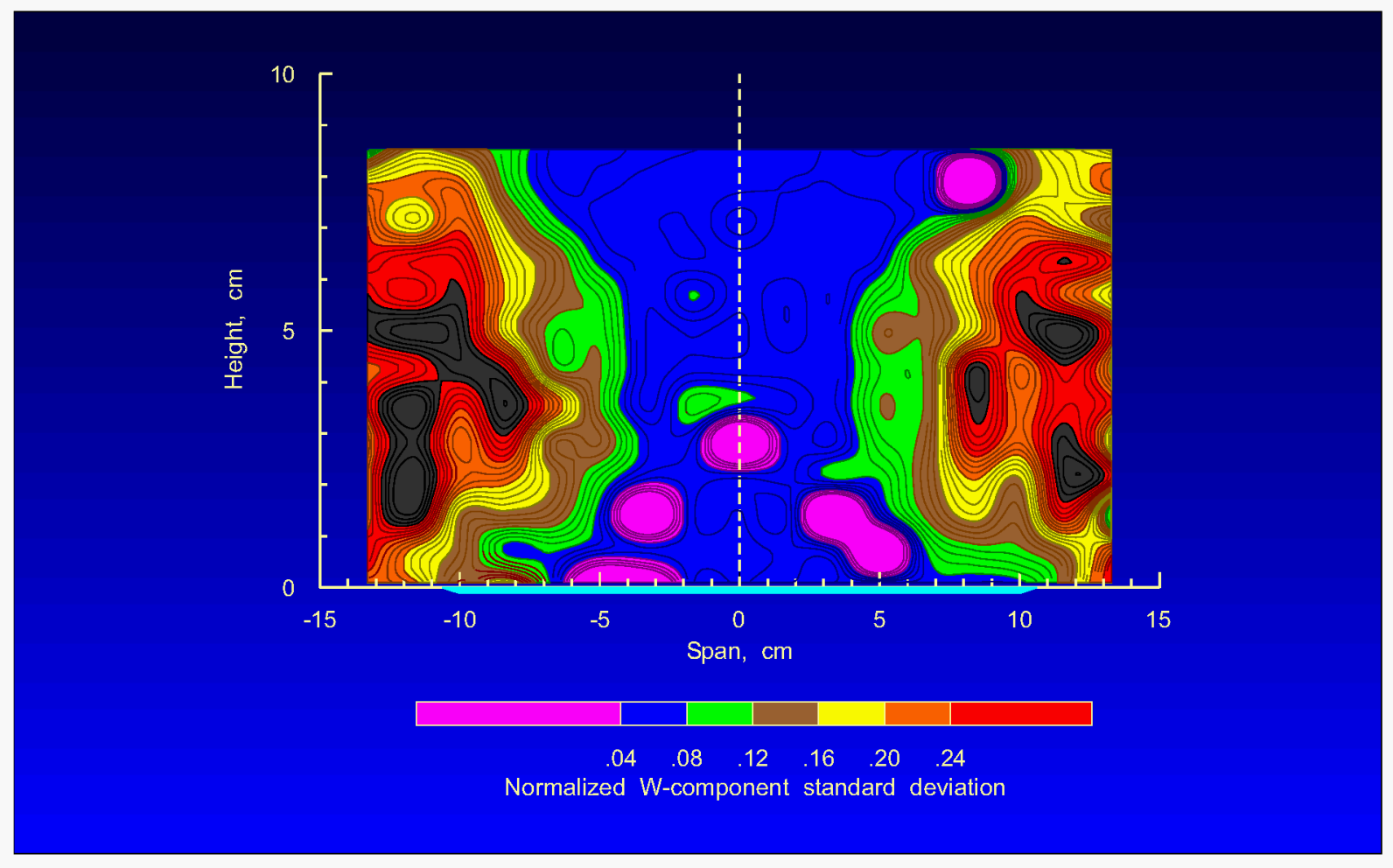

Figure 15.- Contours of traverse turbulence intensity, $\alpha=20.5^{\circ}$.

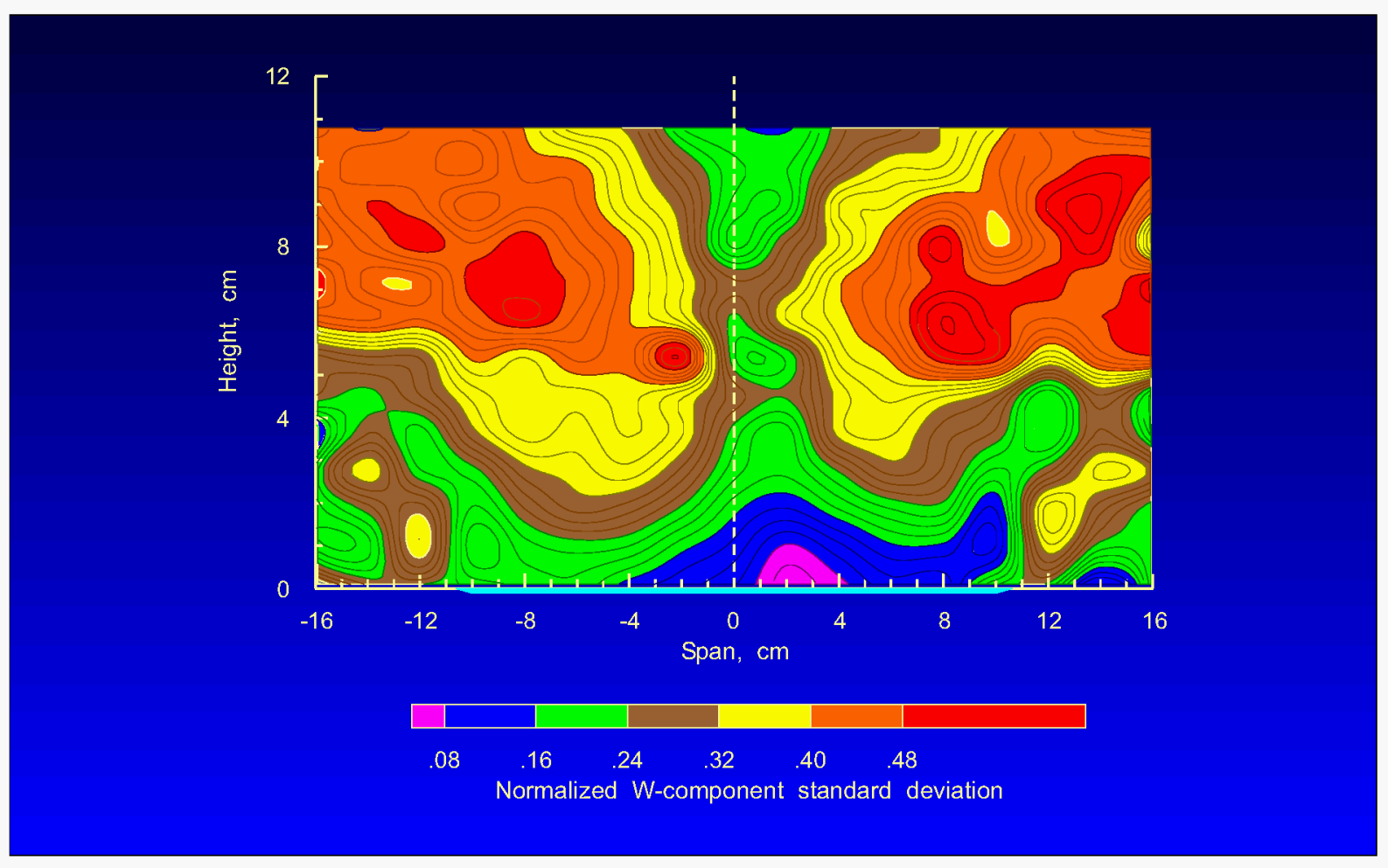

Figure 16.- Contours of traverse turbulence intensity, $\alpha=40.0^{\circ}$. 


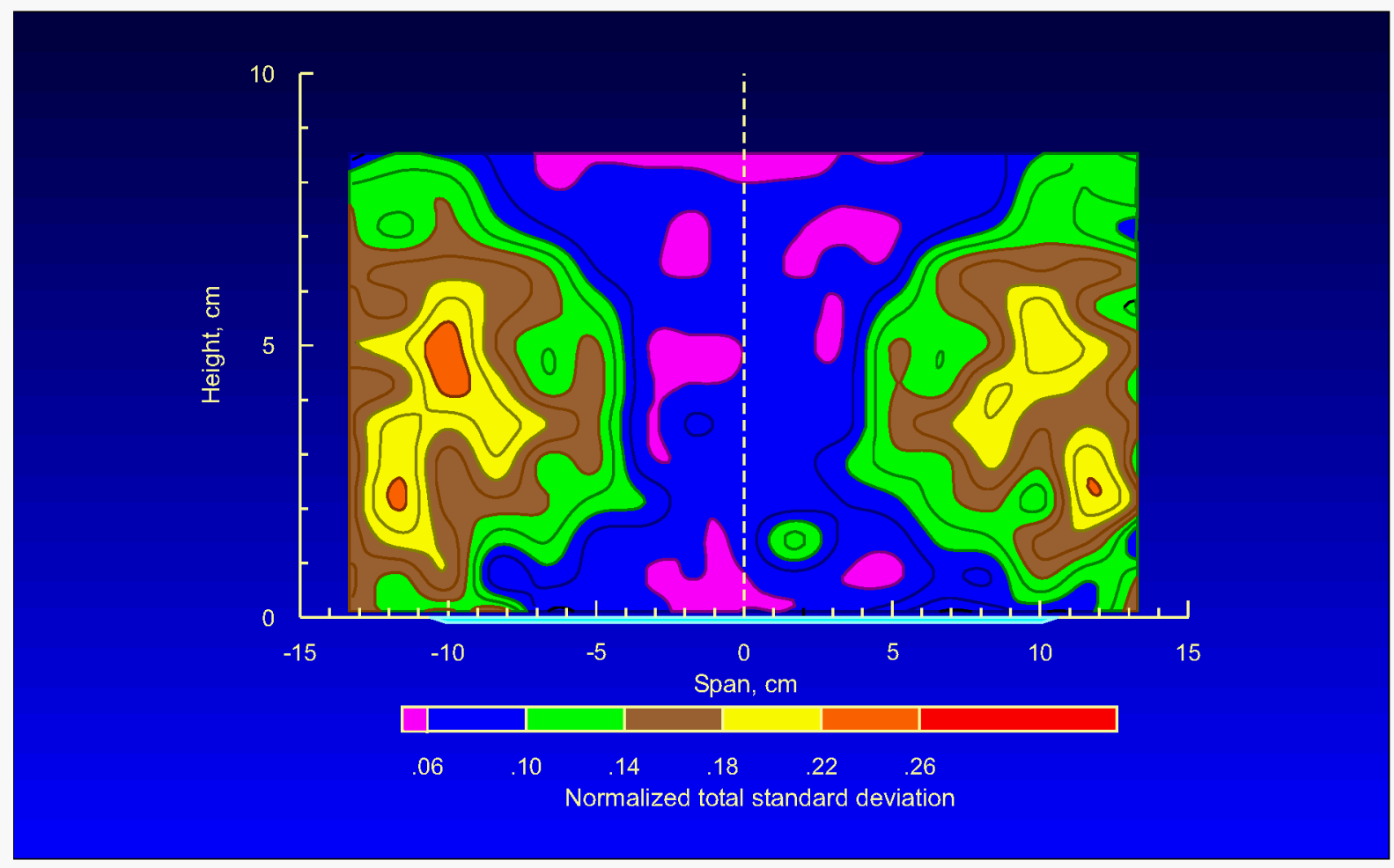

Figure 17.- Contours of estimates of total turbulence intensity, $\alpha=20.5^{\circ}$.

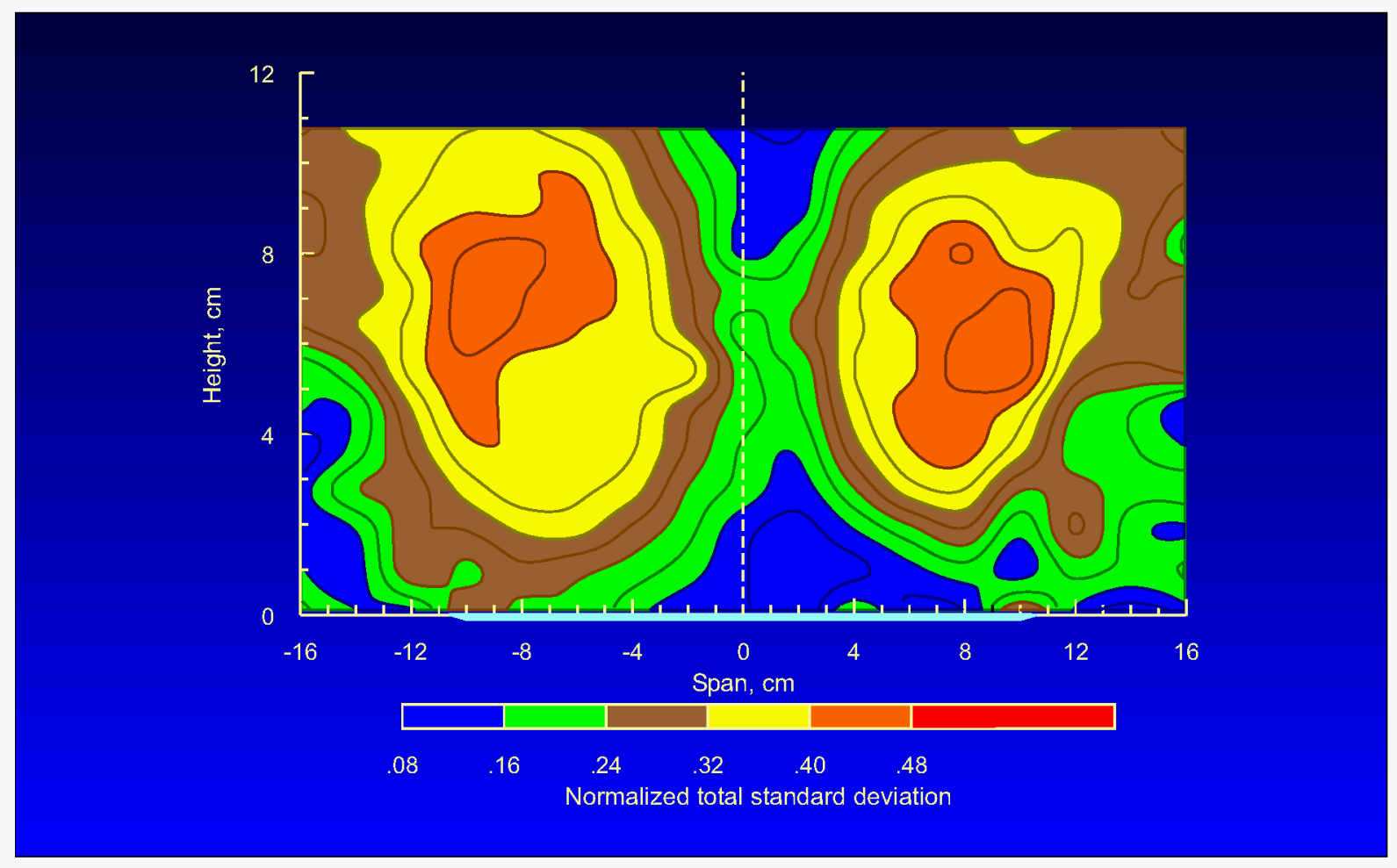

Figure 18.- Contours of estimates of total turbulence intensity, $\alpha=40.0^{\circ}$. 


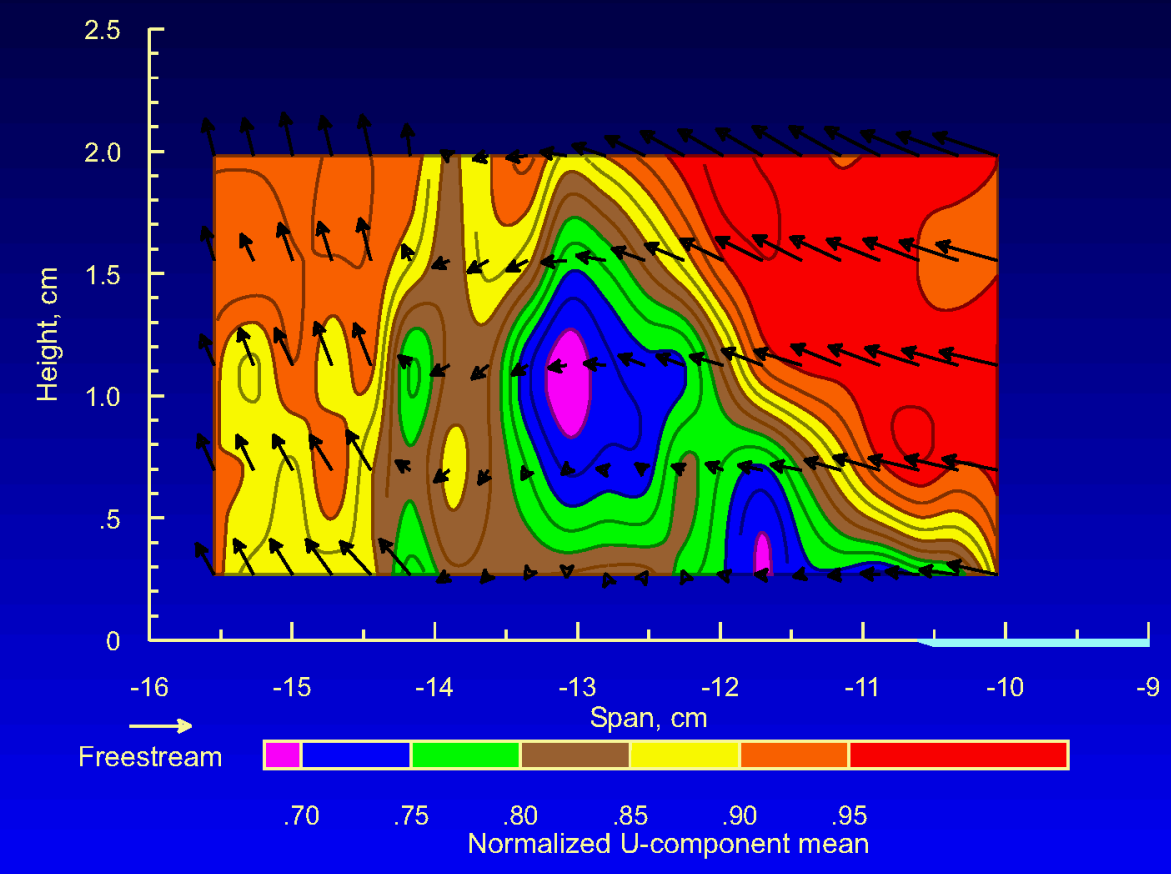

Figure 19.- Mean velocity measurements of a secondary vortex, $\alpha=20.5^{\circ}$ 